programmes to inform the public of the presence and objectives of these groups. As results of the research become well enough developed to warrant it, we hope to organize both technical and semi-popular symposia for discussing them, and to hold these symposia in localities as close as possible to the places where the bulk of the research has been done. At the same time, public lectures on particular phases of the work by biologists having a world-wide reputation would aid both the IBP and the recognition of biology on the part of the general public. More definite plans for this phase of the programme cannot be made until its research phase is more fully planned, but in the long run this type of activity may become one of the most valuable contributions of the programme as a whole.

Standardizing the language of biologists; increasing the opportunities for direct contacts between biologists of different countries, co-ordinated biological research on an international scale, and world-wide stimulation of training and education in biology; these objectives are all broad, timely and worthwhile. Though its means are modest, the International Union of Biological Sciences is doing what it can to fulfil these needs. We are most anxious to welcome into our organization groups of biologists who feel that at this critical period of the Earth's history such objectives are worthy of their attention.

\title{
CHEMISTRY AND MENTAL DISEASE
}

\section{A} JOINT symposium organized by the Royal Institute of Chemistry, the Chemical Society and the Society of Chemical Industry was held on September 26 in the University of Manchester. The purpose of the symposium was to review and discuss the more recent advances, as they affect the chemist, that have been made in the treatment, and in understanding the cause, of mental disease.

During the morning session the chairman was Dr. F. L. Rose (Imperial Chemical Industries, Ltd., Pharmaceuticals Division). He welcomed those present, and proceeded to contrast the half-day devoted to the topic during a similar symposium in 1957 with the present symposium occupying a whole day. This was an indication of the increased tempo of research and of the increasing interest in this particular field of medicine. With the flood of novel chemotherapeutic agents and new biochemical information had come a battery of new tests and indeed almost a new language. While listening to the accounts of the recent developments in the treatment of mental disease, we should not forget the debt owed to earlier workers, nor should we omit to recognize how many of the more recent discoveries were based on earlier drugs and observation of their side-effects.

The opening address was delivered by $\mathrm{Dr}$. A. Todrick (Crichton Royal, Dumfries) who dealt with biochemical processes in relation to mental disease. Since the audience was largely composed of chemists he began his talk by describing a classification of mental disease in which a distinction was drawn between psychosis and neurosis, and between mental deficiency involving an anatomical or biochemical defect present at birth which prevents normal development, and mental illness which is understood to imply a disturbance in previously normal individuals. In recent years a greatly increased research effort, much of the credit for which goes to the pharmaceutical firms, has produced many valuable drugs. On the biochemical side the research effort has been aimed at discovering a biochemical lesion or a biochemical consequence of the lesion which might assist in diagnosis or in control of treatment. A great deal of work has been done on schizophrenia but much less on the manic, depressive psychosis, probably because the latter disease is more capable of being effectively treated. Many hypotheses as to the cause of schizophrenia have been proposed, but most of them involving enzyme or endocrine imbalance, or defects of the liver, central nervous, homeostatic or vascular systems, it was never clear whether the abnormality was a cause of, or a result of, schizophrenia. The body fluids of schizophrenic patients have been subjected to intensive examination both before and after drug administration. Many of the earlier published results were suspect because all too frequently the investigators concerned paid insufficient attention to the effects of drugs being administered at the time the experiments were being performed. The endogenous toxin explanation for the cause of schizophrenia has been popular for a long time, and results both in support of and against the hypothesis have been forthcoming from different laboratories.

Recently, highly significant findings have been obtained by Roman and Malcolm in Aberdeen, who have shown that the induced potentials produced in rat brain by the application of schizophrenic plasmas are clearly distinguishable from those produced by normal plasmas. The next step in the investigation would be to attempt the isolation of the compound responsible for the effect. Based on the views of Brodie that noradrenaline is the hormone responsible for the ergotrophic or activating system in the central nervous system, and that 5-hydroxytryptamine is the hormone responsible for the trophotropic or inhibitory system, the levels of these two agents and their metabolic products in the blood and urine of both normal and schizophrenic patients have been frequently measured and compared. The study of metabolites has been made easier latterly by increased knowledge on the metabolic pathways for the two hormones. Recent work with radioactive serotonin and also estimations of the urinary level of 3-methoxy4-hydroxymandelic acid (a metabolite of noradrenal. ine), has indicated that there is no significant difference between the way and extent to which these two hormones are metabolized by schizophrenic and normal human subjects. The biochemical lesion present in schizophrenia, if indeed such a lesion exists, remains to be identified. Clinical studies with drugs which produce changes in behaviour are also shedding much new light on the problem of mental illness, but the biochemical processes initiated by these agents are only beginning to be understood.

Dr. L. I. Woolf (Department of the Regius Professor of Medicine, Radeliffe Infirmary, Oxford), then spoke about inborn metabolic errors and mental disease, with special reference to the genetic metabolic disorder, phenylketonuria. He was primarily concerned with mental deficiency, which was usually sharply distinguished, particularly by psychologists, 
from mental illness. The difference was perhaps not so great as sometimes supposed, being at least partly related to the age at which the disability becomes apparent. In the mentally deficient the damage to the brain usually commences shortly after birth, and normal intelligence has no time to develop, whereas in the psychotic individual normal intelligence is evident until late childhood or adult life, and is then followed by progressive decline and increasing dementia.

Phenylketonurics are mentally defective, usually having an intelligence quotient of less than 25 , and they are often markedly disturbed. The discase, which become detectable at six months of age and which is pronounced at two years of age, is due to the absence of the pair of genes responsible for synthesizing the enzyme, phenylalanino hydroxylase. Absence of this enzyme results in an accumulation of dietary phenylalanine due to non-conversion to tyrosine. Some of the phenylalanine, which is not readily excreted, is converted to abnormal metabolites which are known to inhibit several decarboxylase enzymes that are important to the normal functioning of the brain. There is also evidonce of the failure to lay down myolin in the brain nerve fibres of phenylketonurics, and/or the destruction of myelin already formed. Treatment consists of a diot free from phenylalanine, which to be effective must be initiated at an early stage in the development of the disease before extensive irreversible damage has occurred. This can now be assured by routine examination of the urine of all new-born babies for the presence of phonyl pyruvic acid. Analytical control is essential and the concentration of phenylalanino in the blood must be measured and adjusted to rotain the minimum roquired for maintonance of life. Other metabolic errors affecting the mind which are due to the congenital absence of essontial enzymes are cretinism caused by an absenco of thyroid hormone, maple syrup urine disease (leucinosis) caused by incomplete amino-acid metabolism, and galactosæmia caused by inability to convert galactose to glycogen.

A paper entitled "The Pharmacological Assessment of Psychotropic Activity", presented by Dr. M. W. Parkes (Roche Products, Ltd.), completed the morn. ing's programme. The relative ease of devising an animal test for bacterial and parasite infoctions, or for organic diseases such as hypertension, in which the animal test bore some relevance to the disease state in man, was contrasted with the difficulties of devising an animal test that in any way resembled human mental illness. It was the development of a higher mental life that distinguished man from the comparatively elementary mental state of all other animals. Yet it is the higher functions of the brain that are affected most seriously by mental illnoss. Nevertheless, psychiatric conditions such as anxiety, aggressiveness, hostility, rago and fear have been set up in animals (following the lines of Pavlov's classical experiments) by the alternate application of reward and punishment. Other techniques involve the electrical stimulation of the brain by electrodes implanted in the hypothalamus, or by exposure to fearful experiences.

The effects of drugs on these conditions have then boen investigated. An entirely empirical approach has been to screen new compounds and to select those showing similar biochemical or pharmacological properties to those of clinically useful drugs even though there may be no apparent connexion between these properties and a property relevant for psycho- therapeutic action. Measurement of the locomotor activity of small rodents has been extensively utilized and a wide variety of methods has been developed for recording and assaying the results. Biochemical techniques have also been invoked, as, for example, the development of a test for antidepressant drugs based on the inhibition in vitro and in vivo of the enzyme monoamine-oxidase. The antagonism between stimulant and sedative drugs has formed the basis of a number of screening procedures, and is almost the only way of selecting drugs likely to be antidepressants of the imipramine and amitryptyline class. The use of hallucinogenic agents or psychomimetics to produce an artificial psychosis has been utilized in human volunteers, but it is of doubtful application in animals because of the difficulty of measuring the effects produced. Further. more, in both man and animals it is questionable whether the psychosis produced is in any way related to schizophrenia. All means of looking for psychotropic activity are undoubtedly empirical to a greater or lesser extent, even where a basis of relovance is apparent. Tranquillizing or antipsychotic properties appear to be best detected by tests depending on the emotional components of animal behaviour.

The chair was occupied during tho afternoon session by Prof. R. N. Haszeldine (Manchester College of Science and Technology). The first speaker, Dr. A. F. Crowther (Imperial Chemical Industries, Ltd., Pharmacouticals Division) dealt with structure activity relationships in the tranquillizer group of drugs. 'Tranquillizers were divided into two types, designated 'major' and 'minor'. Since the milder-acting minor tranquillizers, which are used in the treatment of neuroses, are the most difficult to evaluate clinically - the placebo effect being most marked in their case-structure-activity relationships based on clinical trials are less meaningful than those obtained with the major tranquillizers. The latter, which are used in the treatment of psychotic states can be classified into two main groups depending on whether they are based on the phenothiazine molecule or the Rauwolfia alkaloids. The phenothiazines, which comprise a large compact group, were developed from the early antihistamine drug promothazine. Most of the variations of the original structure which have resulted in clinically useful drugs consist of changes in the length, the branching and the torminal groups of tho so-called 'basic side-chain' attached to the nitrogen atom of the phenothiazine nucleus, and also in the nature of the substituent in the two-position of the nucleus. Replacement of the nucloar nitrogen and/or sulphur atoms by carbon atoms has given the three othor valuable drugs chlorprothixene, imipramine and amitryptylino. The two last-named are not, however, used as tranquillizers, although pharmacologically they have chlorpromazine-like properties, but as antidepressants. Norchlorpromazine and chlorpromazine $N$-oxide, two metabolites of chlorpromazino, resemble the parent agent in activity in animal behavioural tests. In the Rauwolfia alkaloid series the best-known compound is reserpine, which has both sedative and hypotensive properties. Many attempts have been made to dissociate these two actions, and the activity of derivatives of reserpine without the 11-methoxy group, with variation in the 16-ester group and 18-acyloxy group, were discussed by Dr. Crowthor. The drugs tetrabenazine and benzquinamide were somewhat related in structure to reserpine. The former was claimed as being superior 
to reserpine and chlorpromazine in the treatment of psychoses. A series of piperidinobutyrophenones, of which halopiperidol is a member, falls into this category of major tranquillizers. Of the many minor tranquillizers, some like methaminodiazepoxide have unusual chemical structures, but the majority are glycols, and the related carbamoyl derivatives, amino alcohols and amides.

Psychostimulants, which for this paper at least were defined as those drugs used in the treatment of depression, were the subject of the next paper, presented by Dr. E. H. P. Young (Imperial Chemical Industries, Ltd., Pharmaceuticals Division). The structures of some of the earlier phenylethylamine derivatives and the way in which this molecule may be visualized as a component of more recent drugs such as methylphenidate and pipradrol were illustrated. The earlier drugs have now been almost completely superseded by compounds characterized by their ability to inhibit the enzyme monoamine oxidase, and by a group of drugs related to imipramine. The first and most important members of the former class were derivatives of hydrazine, of which some hundreds have been prepared, and which have readily lent themselves to work on structure-activity relationships. Other differently constituted monoamine enzyme inhibitors were certain harmine derivatives, phenylcyclopropylamines, a-alkyltryptamines and benzylamines. The most potent and clinically useful of the antidepressant drugs were the compounds imipramine and amitryptyline, which as indicated earlier were derived from phenothiazine. Their metabolites, the desmethyl compounds, have been found to be more rapidly effective than the parent drugs. Among other ehemical types which have produced antidepressant properties are certain esters of 3-piperidol, but side-effects have limited their clinical usefulness.

The final paper of the symposium was given by Dr. F. A. Jenner (Department of Psychiatry, University of Sheffield). In it he described some of the problems arising from the use of new modern drugs in psychiatric practice. The new drugs presented a great challenge to the clinician if only because of the rate at which they appeared. The need for sophisticated methods of assay, backed by precise statistical assessment, was essential to overcome the placebo response and other extraneous factors such as the environment of the patient and the doctor's own outlook. Many psychiatric patients are talking about their own private world, and it is the task of the psychiatrist to express this personal experience in psychoanalytical language. However, the use of this specialized language itself imposes its own limitations. The diagnostic terms of psychiatry are words not things, and they cannot be reduced to understandable chemical terms. Nevertheless, it cannot be doubted that the chemical architecture of the brain is relevant to psychology, and most interesting is the finding that the metabolism of monoamines, which are localized in specific areas of this organ, is influenced by drugs of known value in psychiatry. Most of the drugs commonly used treat symptoms and not diseases, but yet they can be shown to be effective in 'double-blind' controlled trials. The phenothiazines have been of great value in the management of schizophrenics, while imipramine, its metabolite and monoamineoxidase inhibitors are established as anti-depressive agents. Anxiety is often alleviated by chlordiazepoxide or diazepam.

The value of drugs in the treatment of neurosis is often more difficult to assess. They may give patients sufficient support to recover spontaneously, since they can certainly induce increased tranquillity, but too often they are used by doctors as an alternative to listening to the patient's own story. Psychotherapy might often produce the same or better results than drugs. The psychomimetic drugs are important tools for psychopharmacology but are doubtful additions to the drug cupboard of hospital wards. The most reliable group of psychotropic drugs are the hypnosedatives, of which the barbiturates are best known; but the great contribution of the major tranquillizers or neuroleptics has been their ability to produce tranquillity without sleep. The major tranquillizers frequently produce extra-pyramidal side-effects which can, however, be treated with effective anti-Parkinsonian drugs. The tranquillosedatives such as meprobamate, chlordiazepoxide and diazepam tend to be muscle relaxants and are of particular value in allaying anxiety. For the treatment of depression imipramine, amitryptyline and their metabolites are recommended. The monoamine oxidase inhibitors are also effective, but are often toxic. All these new drugs have made the wards quieter, but their overall effects on patients' ability to return to a normal life are more difficult to assess. Psychopharmacology is here to stay, and what has been so far achieved is probably only a prelude to more effective treatment for the mentally disturbed.

The interest shown by the audience present at this symposium was demonstrated by the number of questions put to the speakers at the end of the morning and afternoon sessions. $\quad$ E. H. P. Young

\section{NEWS and VIEWS}

The Royal Society: Medal Awards

THE following medal awards of the Royal Society have recently been announced:

Copley Medal to Sir Cyril Hinshelwood, Dr. Lee's professor of chemistry in the University of Oxford, for his distinguished researches in the field of chemical kinetics, including the study of biological reaction mochanisms, and for his outstanding contributions to natural philosophy.

Rumford Medal to Prof. D. M. Newitt, emeritus professor of chemical engineering in the University of London, for his distinguished contributions to chemical engineering.
Davy Medal to Prof. H. J. Emeléus, professor of inorganic chemistry in the University of Cambridge, for his distinguished researches in inorganic chemistry and the discovery and examination of a wide range of new compounds.

Darwin Medal to Prof. G. G. Simpson, Alexander Agassiz professor of vertebrate palæontology, Museum of Comparative Zoology, Harvard University, for his distinguished contributions to general evolutionary theory, based on a profound study of palæontology, particularly of vertebrates.

Buchanan Medal to Sir Landsborough Thomson, formerly second secretary of the Medical Research 